\title{
Risk Modelling \\ Planet'S Modern Information Sources for Frequent Status Assessment and Risk Modelling
}

\author{
$\underline{\text { Rene Griesbach }}^{1}$, Vesna Tunguz ${ }^{2}$ \\ ${ }^{1}$ Planet Labs Germany GmbH, 10719 Berlin, Germany \\ ${ }^{2}$ University of East Sarajevo, Faculty of Agriculture, Bosnia and Herzegovina
}

doi: https://doi.org/10.21467/abstracts.93.23

\begin{abstract}
A B S T RA C T
Our Planet faces dramatic changes due to changing climate. It is obvious, that with changing environmental conditions all risk management strategies have to be critically reviewed frequently. But such process needs frequently updated status information about the any of the risk factors and even better, a projection of their status into the future. Today, the COPERNICUS program of the European Union provides valuable Sentinel-2 satellite observation free of charge. This is a huge source of information about our environment and shall be utilized wherever possible. It allows to derive information about vegetation cover, relief conditions, water quality, sun exposure and many more fields of interest with a spatial resolution (pixel size) of $10 \mathrm{~m}$ on the ground every 3-5 days. Such temporal resolution may sometimes not be sufficient, considering the frequent cloud cover in the areas which interest us. If an acquisition date falls on a cloudy day, then the next image can be expected only 3-5 days later, which is then hopefully cloud-free. Sometimes the $10 \mathrm{~m}$ spatial resolution of Sentinel-2 satellite is not sufficient to assess risk factors.

Planet provides a complementing data source for these cases. It's Planet Scope constellation of currently more than 120 satellites images almost the entire landmass of the Earth every single day with a spatial resolution of 3-4 m. These commercially available images can be accessed a few hours after image acquisition via a sophisticated web-based API and/or GUI and complement the available and usable Sentinel-2 images. For even higher requirements on spatial resolution Planet can provide imagery of its SkySat constellation of currently 13 satellites. Twice per day it allows to take a four-band image with a spatial resolution of up to 72 $\mathrm{cm}$ for selected areas. In 2020, 6 more SkySat satellites will be launched and integrated into this constellation. At the same time Planet lowers the orbits of the other SkySat satellites, resulting in a pixel resolution of $0.5 \mathrm{~m}$ and the possibility to image selected areas up to 10 times per day.

The principle and the technological specifications of the Planet constellations and image products are laid out in this presentation. It further focuses on the benefits of such data source for professional risk management. Planet satellite data provides the measurements over periods of time, which is essential to discover trends of developments and changes. These measurements refine and calibrate risk models to predict future conditions of our environment. Only with profound predicted values the correct decisions for risk mitigation can be made.

Several real use-cases from different fields of interest will be presented, among them Oil and Gas, Urban sprawl, Agriculture, Forestry and Environmental Protection. All these benefit from the frequent availability of high and very high resolution multi-spectral imagery from space. Needless to say, how important such operative satellite data collection is for frequent re-assessment of risks in case of disaster situations.

Agriculture is the most vulnerable branch of our economies for climate change induced risks. With the help of monitoring the status of the vegetation cover, information about the soil conditions can be collected with satellites and put into long-term context for risk assessment. Remote Sensing technologies based on satellite imagery are essential to master the challenge of climate change in modern risk management.

(C) 2020 Copyright held by the author(s). Published by AIJR Publisher in "Abstracts of The Second Eurasian RISK-2020 Conference and Symposium” April 12- 19, 2020, Tbilisi, Georgia. Jointly organized by AMIR Technical Services LLC, Georgian Technical University, Institute of Geography (Kazakhstan) and Russian Institute of Petroleum Geology and Geophysics.

AijR DOI: $10.21467 /$ abstracts. 93
\end{abstract}

